\title{
Influence of detector motion in Bell inequalities with entangled fermions
}

\author{
André G. S. Landulfo and George E. A. Matsas \\ Instituto de Física Teórica, Universidade Estadual Paulista, Rua Pamplona 145, 01405-900 São Paulo, SP, Brazil
}

(Received 11 December 2008; published 24 April 2009)

\begin{abstract}
We investigate how relativity influences the spin correlation of entangled fermions described by wave packets and measured by moving detectors. In particular, we show that the Clauser-Horne-Shimony-Holt (CHSH) Bell inequality is not violated by quantum mechanics when the left and right spin detectors move fast enough. We also show that the violation of the Bell-CHSH inequality measured by the moving detectors decreases with increasing width of the initial wave packet.
\end{abstract}

DOI: $10.1103 /$ PhysRevA.79.044103

PACS number(s): 03.65.Ud, 03.30.+p

The discovery of the Bell inequalities can be considered one of the most important physics landmarks of the 20th century [1]. It allows us to probe the essence of quantum theory by distinguishing it from local hidden variable theories. The genesis of this achievement can be traced back to the Einstein-Podolsky-Rosen discussion about the completeness of quantum mechanics [2]. Presently the Clauser-HorneShimony-Holt (CHSH) Bell inequality [3] has been shown to be violated by 30 standard deviations [4], which strongly supports quantum mechanics. In order to contribute to the intense present debate on the interplay between relativity and quantum mechanics (see, e.g., Refs. [5-10]), we investigate here how the former influences the spin correlation of entangled fermions measured by moving detectors. In particular we show that the CHSH Bell inequality can be satisfied rather than violated by quantum mechanics if the left and right spin detectors are set in fast enough relativistic motion. We adopt natural units $\hbar=c=1$.

Let us assume a system composed of two spin-1/2 particles $A$ and $B$ with mass $m$ and zero total spin angular momentum. Each particle spin is measured along some arbitrary direction defined on the $y \perp z$ plane. The distance between the planes along the $x$ axis is large enough to make both measurements causally disconnected. This is well known that local hidden variable theories satisfy the $\mathrm{CHSH}$ Bell inequality

$$
\left|E\left(\mathbf{a}_{2}, \mathbf{b}_{1}\right)+E\left(\mathbf{a}_{2}, \mathbf{b}_{2}\right)+E\left(\mathbf{a}_{1}, \mathbf{b}_{1}\right)-E\left(\mathbf{a}_{1}, \mathbf{b}_{2}\right)\right| \leq 2,
$$

where $\mathbf{a}_{\mathbf{i}}(i=1,2)$ are two arbitrary unit vectors contained in the $y \perp z$ plane along which the spin $\mathbf{s}_{A}$ of particle $A$ is measured, and analogously for the two arbitrary unit vectors $\mathbf{b}_{\mathbf{j}}$ $(j=1,2)$ and spin $\mathbf{s}_{B}$ of particle $B$. Here

$$
E\left(\mathbf{a}_{\mathbf{i}}, \mathbf{b}_{\mathbf{j}}\right) \equiv \lim _{N \rightarrow \infty} \frac{4}{N} \sum_{n=1}^{N}\left(\mathbf{a}_{\mathbf{i}} \cdot \mathbf{s}_{A}\right)\left(\mathbf{b}_{\mathbf{j}} \cdot \mathbf{s}_{B}\right)
$$

is the spin correlation function obtained after an arbitrarily large number $N$ of experiments is performed, and $\mathbf{a}_{\mathbf{i}} \cdot \mathbf{s}_{A}$ and $\mathbf{b}_{\mathbf{j}} \cdot \mathbf{s}_{B}$ assume $\pm 1 / 2$ values.

Let us now test inequality (1) in the context of quantum mechanics, where we allow the left and right detectors to move along the $x$ axis. For this purpose let us begin considering a quantum system composed of two spin-1/2 particles. The corresponding normalized state can be written as $[6,11]$ (see also Ref. [12])

$$
|\psi\rangle=\sum_{s_{A}, s_{B}} \int d \mathbf{p}_{A} d \mathbf{p}_{B} \psi_{s_{A} s_{B}}\left(\mathbf{p}_{A}, \mathbf{p}_{B}\right)\left|s_{A}, p_{A}\right\rangle\left|s_{B}, p_{B}\right\rangle,
$$

where

$$
\begin{gathered}
\sum_{s_{A}, s_{B}} \int d \mathbf{p}_{A} d \mathbf{p}_{B}\left|\psi_{s_{A} s_{B}}\left(\mathbf{p}_{A}, \mathbf{p}_{B}\right)\right|^{2}=1, \\
\left\langle s_{X}^{\prime}, p_{X}^{\prime} \mid s_{X}, p_{X}\right\rangle=\delta_{s_{X}^{\prime} s_{X}} \delta\left(\mathbf{p}_{X}^{\prime}-\mathbf{p}_{X}\right),
\end{gathered}
$$

and $X=A, B$ distinguishes between both particles. Taking $P_{X}^{\mu}$ and $\mathbf{S}_{X} \equiv \mathbf{s}_{X} \otimes I$ as the four-momentum and Wigner spin operators, respectively, where $\mathbf{s}_{X}$ is half of the Pauli matrices, we have

$$
\begin{gathered}
P_{X}^{\mu}\left|s_{X}, p_{X}\right\rangle=p_{X}^{\mu}\left|s_{X}, p_{X}\right\rangle, \\
S_{X}^{z}\left|s_{X}, p_{X}\right\rangle=s_{X}\left|s_{X}, p_{X}\right\rangle,
\end{gathered}
$$

with $p_{X}=\left(\sqrt{\mathbf{p}_{X}^{2}+m^{2}}, \mathbf{p}_{X}\right)$ and $s_{X}= \pm 1 / 2$. Let us now assume that the two-particle system is prepared in a singlet state (see, e.g., Refs. $[9,13]$ for the two-spinor notation used below)

$$
\begin{aligned}
\psi\left(\mathbf{p}_{A}, \mathbf{p}_{B}\right)= & \frac{1}{\sqrt{2}}\left[\left(\begin{array}{c}
f_{\mathbf{k}_{A}}\left(\mathbf{p}_{A}\right) \\
0
\end{array}\right) \otimes\left(\begin{array}{c}
0 \\
f_{\mathbf{k}_{B}}\left(\mathbf{p}_{B}\right)
\end{array}\right)-\left(\begin{array}{c}
0 \\
f_{\mathbf{k}_{A}}\left(\mathbf{p}_{A}\right)
\end{array}\right)\right. \\
& \left.\otimes\left(\begin{array}{c}
f_{\mathbf{k}_{B}}\left(\mathbf{p}_{B}\right) \\
0
\end{array}\right)\right]
\end{aligned}
$$

from which we read

$$
\begin{aligned}
\psi_{s_{A} s_{B}}\left(\mathbf{p}_{A}, \mathbf{p}_{B}\right)= & \frac{1}{\sqrt{2}} f_{\mathbf{k}_{A}}\left(\mathbf{p}_{A}\right) f_{\mathbf{k}_{B}}\left(\mathbf{p}_{B}\right) \\
& \times\left(\delta_{s_{A} 1 / 2} \delta_{s_{B}-1 / 2}-\delta_{s_{A}-1 / 2} \delta_{s_{B} 1 / 2}\right) .
\end{aligned}
$$

We describe particles $A$ and $B$ by Gaussian packets: $f_{\mathbf{k}_{X}}\left(\mathbf{p}_{X}\right)=\pi^{-3 / 4} w^{-3 / 2} e^{-\left(\mathbf{p}_{X}-\mathbf{k}_{X}\right)^{2} /\left(2 w^{2}\right)}, w \in R_{+}$and assume that they move away from the origin in opposite directions at the same rate along the $x$ axis as defined in the laboratory frame: $\mathbf{k}_{A}=-\mathbf{k}_{B}=(|k|, 0,0)$.

Now we are ready to discuss the spin measurement when the detectors acting on particles $A$ and $B$ have velocities $\mathbf{v}_{d_{A}}=\left(v_{d_{A}}, 0,0\right)$ and $\mathbf{v}_{d_{B}}=\left(v_{d_{B}}, 0,0\right)$, respectively. This is im- 
portant to note that each detector will see the wave function $|\psi\rangle$ in their proper frames transformed through an unitary transformation $[11,14]$ :

$$
|\psi\rangle \rightarrow\left|\psi^{\prime}\right\rangle=U\left(\Lambda_{d_{A}}\right) \otimes U\left(\Lambda_{d_{B}}\right)|\psi\rangle,
$$

where

$$
U\left(\Lambda_{d_{X}}\right)\left|s_{X}, p_{X}\right\rangle=\left[\left(\Lambda_{d_{X}} p_{X}\right)^{0} / p_{X}^{0}\right]^{1 / 2} \sum_{s_{X}^{\prime}} D_{s_{X}^{\prime} s_{X}}\left(\Lambda_{d_{X}} p_{X}\right)\left|s_{X}^{\prime}, \Lambda_{d_{X}} p_{X}\right\rangle
$$

The Wigner rotation can be written in matrix form as

$$
\begin{aligned}
D\left(\Lambda_{d_{X}}, p_{X}\right)= & \frac{\left(p_{X}^{0}+m\right) \sigma^{0} \cosh \left(\alpha_{d_{X}} / 2\right)}{\left\{\left(p_{X}^{0}+m\right)\left[\left(\Lambda_{d_{X}} p_{X}\right)^{0}+m\right]\right\}^{1 / 2}} \\
& +\frac{\left(p_{X}^{x} \sigma^{0}+i \epsilon^{x i j} p_{X}^{i} \sigma^{j}\right) \sinh \left(\alpha_{d_{X} / 2}\right)}{\left\{\left(p_{X}^{0}+m\right)\left[\left(\Lambda_{d_{X}} p_{X}\right)^{0}+m\right]\right\}^{1 / 2}}
\end{aligned}
$$

where $\sigma^{0}$ and $\sigma^{i}, i=x, y, z$, are the usual $2 \times 2$ identity and Pauli matrices, respectively. We recall that

$$
\Lambda_{d_{X}}=\left(\begin{array}{cccc}
\cosh \alpha_{d_{X}} & \sinh \alpha_{d_{X}} & 0 & 0 \\
\sinh \alpha_{d_{X}} & \cosh \alpha_{d_{X}} & 0 & 0 \\
0 & 0 & 1 & 0 \\
0 & 0 & 0 & 1
\end{array}\right),
$$

with $\alpha_{d_{X}} \equiv-\tanh ^{-1} v_{d_{X}}$. By using Eqs. (3) and (9) in Eq. (8), we obtain

$$
\begin{aligned}
\left|\psi^{\prime}\right\rangle= & \sum_{s_{X}, s_{X}^{\prime}} \int d \mathbf{p}_{A} d \mathbf{p}_{B}\left(\frac{\left(\Lambda_{d_{A}}^{-1} p_{A}\right)^{0}}{p_{A}^{0}}\right)^{1 / 2}\left(\frac{\left(\Lambda_{d_{B}}^{-1} p_{B}\right)^{0}}{p_{B}^{0}}\right)^{1 / 2} \\
& \times D_{s_{A}^{\prime} s_{A}}\left(\Lambda_{d_{A}}, \Lambda_{d_{A}}^{-1} p_{A}\right) D_{s_{B}^{\prime} s_{B}}\left(\Lambda_{d_{B}}, \Lambda_{d_{B}}^{-1} p_{B}\right) \\
& \times \psi_{s_{A} s_{B}}\left(\Lambda_{d_{A}}^{-1} \mathbf{p}_{A}, \Lambda_{d_{B}}^{-1} \mathbf{p}_{B}\right)\left|s_{A}^{\prime}, p_{A}\right\rangle\left|s_{B}^{\prime}, p_{B}\right\rangle
\end{aligned}
$$

where we have performed the change of variable $p_{X}$ $\rightarrow \Lambda_{d_{X}}^{-1} p_{X}$ and we recall that $d \mathbf{p}_{X} / p_{X}^{0}$ is a relativistic invariant. By using Eqs. (7) and (10) in Eq. (11), we can write $\psi_{s_{A} s_{B}}^{\prime}\left(\mathbf{p}_{A}, \mathbf{p}_{B}\right)$, which appears in

$$
\left|\psi^{\prime}\right\rangle=\sum_{s_{A}, s_{B}} \int d \mathbf{p}_{A} d \mathbf{p}_{B} \psi_{s_{A} s_{B}}^{\prime}\left(\mathbf{p}_{A}, \mathbf{p}_{B}\right)\left|s_{A}, p_{A}\right\rangle\left|s_{B}, p_{B}\right\rangle
$$

using the two-spinor notation:

$$
\begin{aligned}
\psi^{\prime}\left(\mathbf{p}_{A}, \mathbf{p}_{B}\right)= & \frac{1}{\sqrt{2}}\left[\left(\begin{array}{c}
a_{1}\left(\mathbf{p}_{A}\right) \\
a_{2}\left(\mathbf{p}_{A}\right)
\end{array}\right) \otimes\left(\begin{array}{l}
b_{1}\left(\mathbf{p}_{B}\right) \\
b_{2}\left(\mathbf{p}_{B}\right)
\end{array}\right)-\left(\begin{array}{c}
-a_{2}\left(\mathbf{p}_{A}\right) \\
\overline{a_{1}}\left(\mathbf{p}_{A}\right)
\end{array}\right)\right. \\
& \left.\otimes\left(\begin{array}{c}
\overline{b_{2}}\left(\mathbf{p}_{B}\right) \\
-b_{1}\left(\mathbf{p}_{B}\right)
\end{array}\right)\right] .
\end{aligned}
$$

This is the wave function on which the detectors will effectively act to measure the particle spin. Here

$$
\begin{gathered}
a_{1}\left(\mathbf{p}_{A}\right)=K_{A} f_{\mathbf{k}_{A}}\left(\mathbf{q}_{A}\right)\left[C_{A}\left(q_{A}^{0}+m\right)+S_{A}\left(q_{A}^{x}+i q_{A}^{y}\right)\right], \\
a_{2}\left(\mathbf{p}_{A}\right)=K_{A} f_{\mathbf{k}_{A}}\left(\mathbf{q}_{A}\right) S_{A} q_{A}^{z},
\end{gathered}
$$

$$
\begin{gathered}
b_{1}\left(\mathbf{p}_{B}\right)=-K_{B} f_{\mathbf{k}_{B}}\left(\mathbf{q}_{B}\right) S_{B} q_{B}^{z}, \\
b_{2}\left(\mathbf{p}_{B}\right)=K_{B} f_{\mathbf{k}_{B}}\left(\mathbf{q}_{B}\right)\left[C_{B}\left(q_{B}^{0}+m\right)+S_{B}\left(q_{B}^{x}-i q_{B}^{y}\right)\right],
\end{gathered}
$$

where

$$
\begin{gathered}
K_{X} \equiv\left(q_{X}^{0} / p_{X}^{0}\right)^{1 / 2} /\left[\left(q_{X}^{0}+m\right)\left(p_{X}^{0}+m\right)\right]^{1 / 2}, \\
q_{X} \equiv \Lambda_{d_{X}}^{-1} p_{X} \\
C_{X} \equiv \cosh \left(\alpha_{d_{X}} / 2\right) \\
S_{X} \equiv \sinh \left(\alpha_{d_{X}} / 2\right) .
\end{gathered}
$$

Next we trace out the momenta degrees of freedom since the detectors do only measure spin. As a result, we obtain the following reduced density matrix:

$$
\begin{aligned}
\tau^{\prime} & =\int d \mathbf{p}_{A} d \mathbf{p}_{B} \psi^{\prime}\left(\mathbf{p}_{A}, \mathbf{p}_{B}\right) \psi^{\prime \dagger}\left(\mathbf{p}_{A}, \mathbf{p}_{B}\right) \\
& =\left(\rho_{1} \otimes \rho_{1}^{\prime}-\rho_{2} \otimes \rho_{2}^{\prime}-\rho_{3} \otimes \rho_{3}^{\prime}+\rho_{4} \otimes \rho_{4}^{\prime}\right) / 2
\end{aligned}
$$

with

$$
\begin{gathered}
\rho_{1} \otimes \rho_{1}^{\prime}=\left(\begin{array}{cc}
1-V & 0 \\
0 & V
\end{array}\right) \otimes\left(\begin{array}{cc}
W & 0 \\
0 & 1-W
\end{array}\right), \\
\rho_{2} \otimes \rho_{2}^{\prime}=\left(\begin{array}{cc}
0 & 1-3 V \\
-V & 0
\end{array}\right) \otimes\left(\begin{array}{cc}
0 & -W \\
1-3 W & 0
\end{array}\right), \\
\rho_{3} \otimes \rho_{3}^{\prime}=\left(\begin{array}{cc}
0 & -V \\
1-3 V & 0
\end{array}\right) \otimes\left(\begin{array}{cc}
0 & 1-3 W \\
-W & 0
\end{array}\right), \\
\rho_{4} \otimes \rho_{4}^{\prime}=\left(\begin{array}{cc}
V & 0 \\
0 & 1-V
\end{array}\right) \otimes\left(\begin{array}{cc}
1-W & 0 \\
0 & W
\end{array}\right),
\end{gathered}
$$

and

$$
\begin{aligned}
& V\left(\alpha_{d_{A}}\right)=\sinh ^{2}\left(\frac{\alpha_{d_{A}}}{2}\right) \int d \mathbf{q}_{A} \frac{\left|f_{\mathbf{k}_{A}}\left(\mathbf{q}_{A}\right)\right|^{2} q_{A}^{z 2}}{\left(q_{A}^{0}+m\right)\left(p_{A}^{0}+m\right)}, \\
& W\left(\alpha_{d_{B}}\right)=\sinh ^{2}\left(\frac{\alpha_{d_{B}}}{2}\right) \int d \mathbf{q}_{B} \frac{\left|f_{\mathbf{k}_{B}}\left(\mathbf{q}_{B}\right)\right|^{2} q_{B}^{z 2}}{\left(q_{B}^{0}+m\right)\left(p_{B}^{0}+m\right)},
\end{aligned}
$$

where we have used that $d \mathbf{p}_{X} / p_{X}^{0}=d \mathbf{q}_{X} / q_{X}^{0}$. Now let us use our previous results to investigate Eq. (1). In quantum mechanical terms, the left-hand side of this equation can be expressed as

$$
\left|E\left(\mathbf{a}_{2}, \mathbf{b}_{1}\right)+E\left(\mathbf{a}_{2}, \mathbf{b}_{2}\right)+E\left(\mathbf{a}_{1}, \mathbf{b}_{1}\right)-E\left(\mathbf{a}_{1}, \mathbf{b}_{2}\right)\right|=\left|\langle C\rangle_{\tau^{\prime}}\right|,
$$

where $\langle C\rangle_{\tau^{\prime}}=\operatorname{tr}\left(\tau^{\prime} C\right)$ and

$$
C=\left(\sigma \cdot \mathbf{a}_{2}\right) \otimes\left[\sigma \cdot\left(\mathbf{b}_{1}+\mathbf{b}_{2}\right)\right]+\left(\sigma \cdot \mathbf{a}_{1}\right) \otimes\left[\sigma \cdot\left(\mathbf{b}_{1}-\mathbf{b}_{2}\right)\right] .
$$

By using that

$$
\langle(\sigma \cdot \mathbf{u}) \otimes(\sigma \cdot \mathbf{v})\rangle_{\tau^{\prime}}=-(1-2 V)(1-2 W) \mathbf{u} \cdot \mathbf{v},
$$

where $\mathbf{u}, \mathbf{v}=\mathbf{a}_{1}, \mathbf{a}_{2}, \mathbf{b}_{1}, \mathbf{b}_{2}$, we cast Eq. (17) as 

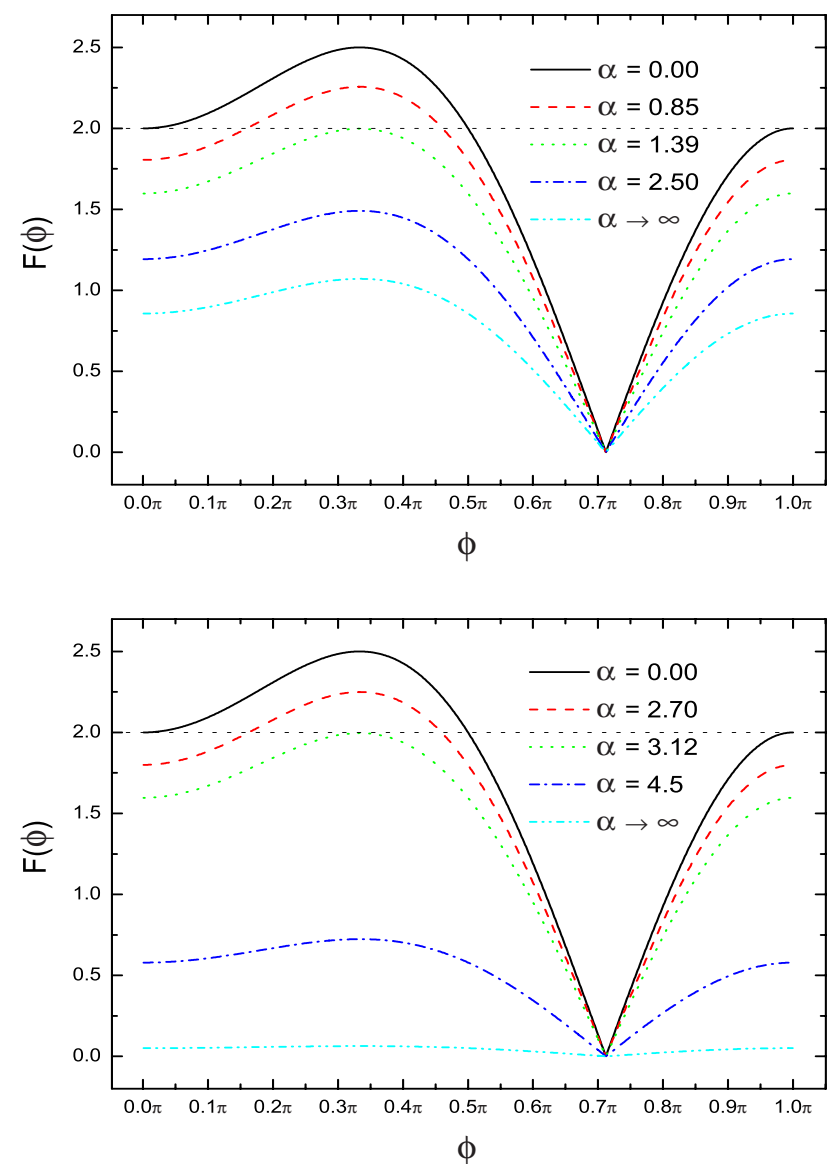

FIG. 1. (Color online) $F(\phi)$ as given in Eq. (22) is plotted as a function of $\phi$ with $\widetilde{w}=4$ for different values of $\alpha$. The top and bottom plots assume $|\widetilde{k}|=0.01$ and $|\widetilde{k}|=100$, respectively. For $\alpha=0$ the usual Bell inequality result is recovered. For $\alpha \gtrsim 1.39$, and $\alpha$ $\geq 3.12$, we have that $F(\phi)<2$ for the top and bottom plots, respectively.

$$
\left|\langle C\rangle_{\tau^{\prime}}\right|=(1-2 V)(1-2 W)\left|\langle C\rangle_{\tau^{\prime}}^{0}\right|
$$

with $\left|\langle C\rangle_{\tau^{\prime}}^{0}\right|=\left|\mathbf{a}_{2} \cdot \mathbf{b}_{1}+\mathbf{a}_{2} \cdot \mathbf{b}_{2}+\mathbf{a}_{1} \cdot \mathbf{b}_{1}-\mathbf{a}_{1} \cdot \mathbf{b}_{2}\right|$. Equation (19) is our key formula. Note that when the detectors are at rest $\alpha_{d_{A}}=\alpha_{d_{B}}=0$, we recover the usual result: $\left|\langle C\rangle_{\tau^{\prime}}\right|=\left|\langle C\rangle_{\tau^{\prime}}^{0}\right|$, i.e., the nontriviality introduced by the detector motion is isolated in the $(1-2 V)(1-2 W)$ multiplicative factor. By defining $\alpha_{i}$, $\beta_{i}(i=1,2)$ as the angles between $\mathbf{a}_{i}, \mathbf{b}_{i}$ and the $y$ axis, respectively, we get

$$
\begin{aligned}
\left|\langle C\rangle_{\tau^{\prime}}^{0}\right|= & \mid \cos \left(\alpha_{2}-\beta_{1}\right)+\cos \left(\alpha_{2}-\beta_{2}\right)+\cos \left(\alpha_{1}-\beta_{1}\right) \\
& -\cos \left(\alpha_{1}-\beta_{2}\right) \mid .
\end{aligned}
$$

For our purposes, this is sufficient to take the simpler case where $\mathbf{a}_{2}=\mathbf{b}_{1}$. By assuming this and $\phi \equiv \cos ^{-1}\left(\mathbf{a}_{1} \cdot \mathbf{a}_{2}\right)$ $=\cos ^{-1}\left(\mathbf{b}_{1} \cdot \mathbf{b}_{2}\right)$, we obtain

$$
\left|\langle C\rangle_{\tau^{\prime}}\right|_{\mathbf{a}_{\mathbf{2}}=\mathbf{b}_{\mathbf{1}}}=(1-2 V)(1-2 W)|1+2 \cos \phi-\cos (2 \phi)| .
$$

Let us now focus on the case where both detectors boost away from each other with the same absolute rapidity [15]: $\alpha_{d_{A}}=-\alpha_{d_{B}}=-|\alpha|$, i.e., $v_{d_{A}}=-v_{d_{B}}=\tanh |\alpha|$. (For $|\widetilde{k}| \ll 1$ we
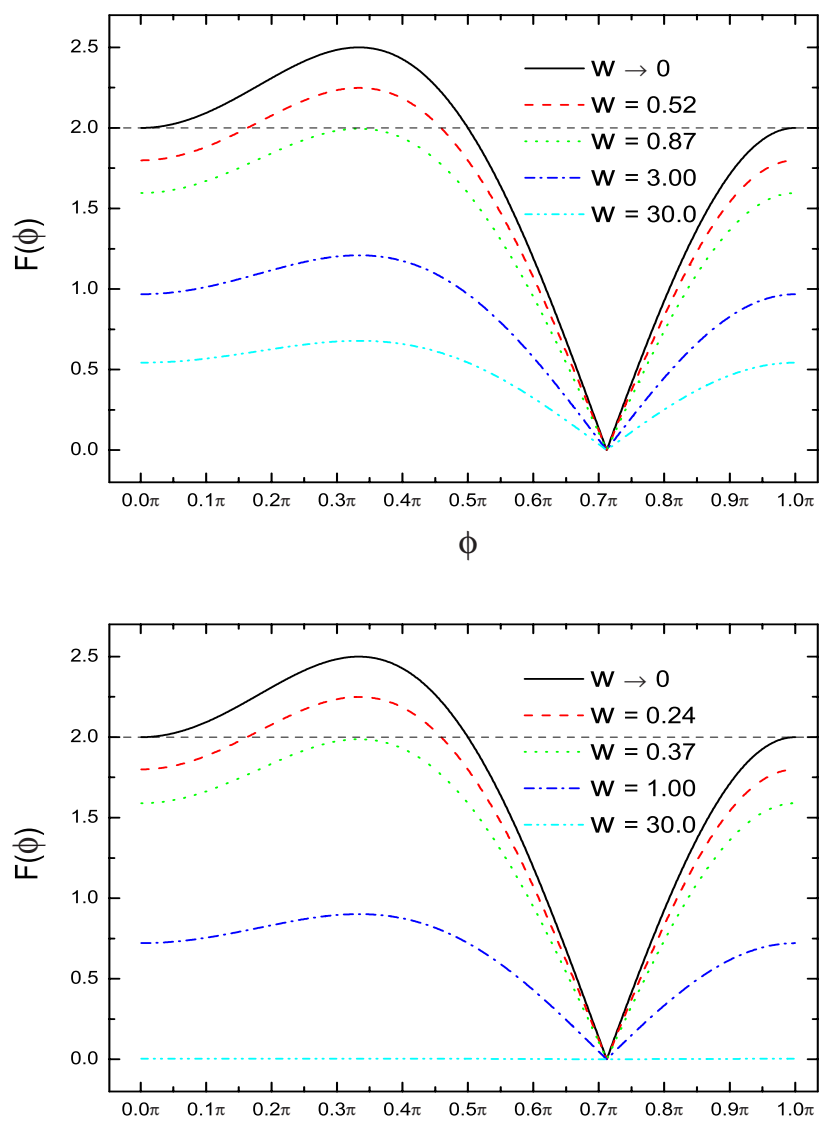

$\phi$

FIG. 2. (Color online) $F(\phi)$ as given in Eq. (22) is plotted as a function of $\phi$ assuming $\alpha \rightarrow \infty$ for different values of the $\widetilde{w}$ width. The top and bottom plots assume again $|\widetilde{k}|=0.01$ and $|\widetilde{k}|=100$, respectively. For $\tilde{w}=0$ the usual Bell inequality result is recovered, while for $\tilde{w} \gtrsim 0.87$ and $\widetilde{w} \gtrsim 0.37$ we have that $F(\phi)<2$ for the top and bottom plots, respectively.

have verified that similar results are obtained no matter if the detectors move away or approach each other, as it should be.) For the sake of simplicity, we define

$$
F(\phi) \equiv\left|\langle C\rangle_{\tau^{\prime}}\right|_{\mathbf{a}_{\mathbf{2}}=\mathbf{b}_{\mathbf{1}}}^{\alpha_{d_{A}}=-\alpha_{d_{B}}}
$$

Then, from Eqs. (15) and (16) we have

$$
V(-|\alpha|)=W(|\alpha|)=\frac{\sinh ^{2}(|\alpha| / 2)}{\sqrt{\pi} \widetilde{w}^{3}} \int_{-\infty}^{\infty} d \widetilde{q}^{x} \int_{0}^{\infty} d \widetilde{q}^{r} G\left(\widetilde{q}^{x}, \widetilde{q}^{r}\right),
$$

where we have used cylindrical coordinates with $q^{x}$ as the symmetry axis and

$$
G\left(\widetilde{q}^{x}, \widetilde{q}^{r}\right)=\frac{\left(\widetilde{q}^{r}\right)^{3} \exp \left\{-\left[\left(\widetilde{q}^{x}-|\widetilde{k}|\right)^{2}+\left(\widetilde{q}^{r}\right)^{2}\right] / \widetilde{w}^{2}\right\}}{\left(\widetilde{q}^{0}+1\right)\left(\widetilde{q}^{0} \cosh |\alpha|-\widetilde{q}^{x} \sinh |\alpha|+1\right)},
$$

with $\widetilde{q}^{r}=q^{r} / m, \widetilde{q}^{x}=q^{x} / m, \widetilde{q}^{0}=\sqrt{\left(\widetilde{q}^{x}\right)^{2}+\left(\widetilde{q}^{r}\right)^{2}+1},|\widetilde{k}|=|k| / m$ and $\tilde{w}=w / m$. Then, we finally obtain the simple expression 


$$
F(\phi)=F_{0}|1+2 \cos \phi-\cos (2 \phi)|,
$$

where $F_{0}=[1-2 V(-|\alpha|)]^{2}$. For very narrow wave packets in the momentum space, i.e., $\widetilde{w} \ll 1$, this is easy to analytically solve the integral in Eq. (20) for particles moving slow enough, $\tilde{k} \approx 0$, and cast Eq. (22) as

$$
\left.F(\phi)\right|_{\tilde{w} \ll 1} ^{\tilde{k} \approx 0}=\left(1-\frac{\widetilde{w}^{2}}{4} \tanh ^{2} \frac{|\alpha|}{2}\right)^{2}|1+2 \cos \phi-\cos (2 \phi)| .
$$

Clearly for $\widetilde{w} \rightarrow 0$, we recover the standard Bell inequality result irrespective of the detector velocities, i.e., the nontriviality driven by the detector motion in Eq. (23) is not present when the entangled particles are described by momenta eigenstates $[8,10]$. This is so because only when particles are described by wave packets, $|\psi\rangle$ (which is a pure state according to observers lying at rest in the laboratory) looks like as a mixed state for the moving detectors once they ignore the momenta degrees of freedom [6]. (The corresponding "missing information" gets hidden in the traced out momenta.)

In Fig. 1 we plot $F(\phi)$ for different detector velocities, i.e., $|\alpha|$ 's, assuming a wave packet with $\tilde{w}=4$. The plots on the top and at the bottom take $|\widetilde{k}|=0.01$ and $|\widetilde{k}|=100$, respectively. We note that the standard Bell inequality result is recovered for $\alpha=0$ but this is not so when the detectors move. Indeed, $F(\phi)$ decreases as the detector velocities increase. For $\alpha \gtrsim 1.39$, and $\alpha \gtrsim 3.12$, we have that $F(\phi)<2$ in the whole $\phi$ range for the $|\tilde{k}|=0.01$ and $|\widetilde{k}|=100$ cases, respectively, i.e., for these $\alpha$ intervals the Bell inequality is not violated for every $\phi$. Our numerical integration was cross checked against the Monte Carlo method and we have verified that it reproduces the analytic value given by Eq. (23) up to 1 part in $10^{5}$. Next we analyze how the packet width influences in our results. In Fig. 2 we plot $F(\phi)$ for different $\widetilde{w}$ values when the detectors have ultrarelativistic velocities: $\alpha \rightarrow \infty$. Again, we have assumed $|\widetilde{k}|=0.01$ and $|\widetilde{k}|=100$ for the top and bottom graphs, respectively. We see that for $\widetilde{w}$ $\rightarrow 0$ we recover the usual Bell inequality result but as the wave packet width $\widetilde{w}$ increases, $F(\phi)$ decreases. This reflects that the nontriviality associated with the detector motion is not manifest when the entangled particles are described by momenta eigenstates. For $\widetilde{w} \gtrsim 0.87$ and $\widetilde{w} \gtrsim 0.37$ we have that $F(\phi)<2$ in the whole $\phi$ range for the top and bottom plots, respectively.

Some experimental effort to verify the influence of the detector motion in the Bell inequalities using photons can be found in the literature [5]. The natural generalization of our results for photons would require the entanglement of horizontal/vertical polarized photons, which is distinct from the one considered in Ref. [5]. This makes both results quite difficult to compare. Furthermore the replacement of fermions by photons is not straightforward [7]. Experiments testing the Bell inequalities for fermions have been recently performed $[16,17]$. The most challenging feature to test our proposal, thus, consists in boosting the detectors giving them high velocities. This can be probably circumvented, however, by keeping the detectors at rest and boosting the states accordingly: $|\psi\rangle \rightarrow\left|\psi^{\prime}\right\rangle=U\left(\Lambda_{d_{A}}\right) \otimes U\left(\Lambda_{d_{B}}\right)|\psi\rangle$ as defined in Eq. (8). It would be interesting to verify our results in laboratory since this would also be an indirect test for all the underlying theoretical framework.

Modern physics is dominated by quantum mechanics and relativity. This is fair to say that the Bell inequalities probe one of the deepest aspects of quantum mechanics. Our analysis shows that the detector state of motion is crucial as one investigates the spin correlation of entangled fermions in the context of the Bell inequalities once one assumes the realistic physical situation where the particles of the entangled system are described by wave packets rather than by momentum eigenstates.

We are grateful to A. O. Pereira for computational assistance. A.L. and G.M. acknowledge full and partial support from Fundação de Amparo à Pesquisa do Estado de São Paulo, respectively. G.M. also acknowledges partial support from Conselho Nacional de Desenvolvimento Científico e Tecnológico.
[1] J. S. Bell, Physics 1, 195 (1964).

[2] A. Einstein, B. Podolsky, and N. Rosen, Phys. Rev. 47, 777 (1935).

[3] J. F. Clauser, M. A. Horne, A. Shimony, and R. A. Holt, Phys. Rev. Lett. 23, 880 (1969).

[4] G. Weihs, T. Jennewein, C. Simon, H. Weinfurter, and A. Zeilinger, Phys. Rev. Lett. 81, 5039 (1998).

[5] A. Stefanov, H. Zbinden, N. Gisin, and A. Suarez, Phys. Rev. Lett. 88, 120404 (2002).

[6] A. Peres, P. F. Scudo, and D. R. Terno, Phys. Rev. Lett. 88, 230402 (2002).

[7] A. Peres and D. R. Terno, J. Mod. Opt. 50, 1165 (2003).

[8] H. Terashima and M. Ueda, Quantum Inf. Comput. 3, 224 (2003).

[9] A. Peres and D. R. Terno, Rev. Mod. Phys. 76, 93 (2004).

[10] W. T. Kim and E. J. Son, Phys. Rev. A 71, 014102 (2005).

[11] F. R. Halpern, Special Relativity and Quantum Mechanics
(Prentice-Hall, Englewood Cliffs, NJ, 1968).

[12] We are adopting Halpern [11] and Peres [6] convention, where the relativistic factor $\sqrt{\mathbf{p}^{2}+m^{2}}$ is introduced further in the Wigner transformation law rather than in the normalization relation.

[13] N. N. Bogolubov, A. A. Logunov, and I. T. Todorov, Introduction to Axiomatic Quantum Field Theory (W. A. Benjamin, Massachusetts, 1975).

[14] S. Weinberg, The Quantum Theory of Fields (Cambridge University Press, Cambridge, 1996), Vol. I.

[15] Similar conclusions are obtained when both detectors are boosted to the same direction.

[16] M. A. Rowe, D. Kielpinski, V. Meyer, C. A. Sackett, W. M. Itano, C. Monroe, and D. J. Wineland, Nature (London) 409, 791 (2001).

[17] H. Sakai et al., Phys. Rev. Lett. 97, 150405 (2006). 